\title{
Revisiting Hardin's parameters for the quantification of particle breakage - A statistical entropy approach
}

\author{
James Leak ${ }^{1}$, Daniel Barreto ${ }^{1 *}$, Vasiliki Dimitriadi ${ }^{1}$, and Emoke Imre $^{2}$ \\ ${ }^{1}$ Edinburgh Napier University, School of Engineering and the Built Environment, Merchiston Campus, Edinburgh, EH10 5DT, U.K. \\ ${ }^{2}$ Óbuda University, EKIK HBM Systems Research Center, 1034 Bécsi út 96/B, Budapest, Hungary.
}

\begin{abstract}
It is well recognised that particle breakage in granular materials is affected by stress level, stress path, initial density, and particle size distribution (PSD), amongst others. Furthermore, it has been shown that breakage has a significant influence on the stress-strain behaviour of soils. This paper compares a commonly used breakage parameter with grading entropy coordinates. Such coordinates enable for the representation of any PSD as a single point in a Cartesian coordinate plane. Hence, the evolution of PSD changes may be easily tracked. This paper aims to demonstrate that grading entropy coordinates are as (or more) effective than other breakage parameters, whilst providing additional insight. On the basis of limited data it is shown that grading entropy coordinates are able to capture the dependence of breakage on stress level, stress path and initial PSD.
\end{abstract}

\section{Introduction}

Critical state behaviour of granular materials under shear is well established. At relatively large strains, granular materials converge to a state of constant volume and stress. Furthermore, stress and volume states define a critical state line (CSL), underpinning the fundamental framework of most existing constitutive modelling approaches [e.g. 1-3].

A key aspect in some of these critical state models is the inclusion of grading-dependent parameters, as it is well recognised that particle crushing affects the behaviour of granular materials. The crushing of particles when subject to stress produces changes in particle size distribution (PSD) that in turn affect the behaviour of soils during compression [4], and the location and/or inclination of the CSL [5-7]. Within this context, parameters to quantify the evolution of the PSD and to predict the amount of particle breakage produced under certain stress conditions are often used [8-10]. These include (i) breakage potential $\left(B_{p}\right)$, and total $\left(B_{t}\right)$ and relative $\left(B_{r}\right)$ breakage, respectively [8]; (ii) breakage internal variable $(B)[9]$; and (iii) grading state index $\left(I_{G}\right)$ [10], amongst others.

A mathematical approach derived from statistical entropy principles has been proposed. The grading entropy coordinates may be used to quantify breakage evolution $[11,12]$ and relate to other geotechnical phenomena such as internal stability [13], liquefaction [14], and dissolution effects [15], amongst others.

Here, Hardin's breakage parameters [8] are examined within the context of the grading entropy coordinates. This is part of a larger study examining the suitability of common PSD descriptors to quantify the effects of grading on various geomechanical properties.
The aim of this paper is to demonstrate that alternative PSD descriptors may be able to provide further insight into granular material behaviour. In particular, estimates about particle breakage and the quantification of its evolution. Whilst our analyses focus on Hardin's parameters, similar comparisons could be made with other existing breakage parameters. Assessing their suitability and effectiveness, however, is out of the scope of this paper. The focus is on grading entropy coordinates, as an alternative PSD descriptor from which further insights could be gained.

\section{Breakage parameters}

In order to understand the stress-strain response of granular materials, it is important to quantify the amount of breakage. As discussed by Hardin [8], this depends on PSD, particle shape, stress state, density, moisture content, as well as the physical characteristics of individual particles. In this study, some of the data presented by Hardin [8] are examined using the grading entropy coordinates defined by Lörincz [11]. In order to assess their suitability to quantify grading changes. Whilst the use of entropy coordinates to quantify particle breakage is not new, comparisons with more commonly used parameters are not available. Additionally, providing such comparison enables any potential advantages/disadvantages to be highlighted; and to provide further insight into phenomena related to particle breakage. Using grading entropy coordinates, the breakage occurring in various size fractions of the material can be assessed, something that cannot be directly performed with other grading parameters. Importantly, as discussed in [8], the main challenge

* Corresponding author: d.barreto@ napier.ac.uk

A video is available at https://doi.org/10.48448/96y9-w622 
when defining measures to adequately quantify particle breakage is to effectively integrate the breakage occurring in the various size fractions of the material.

\subsection{Breakage potential, relative breakage and total breakage - Hardin (1985)}
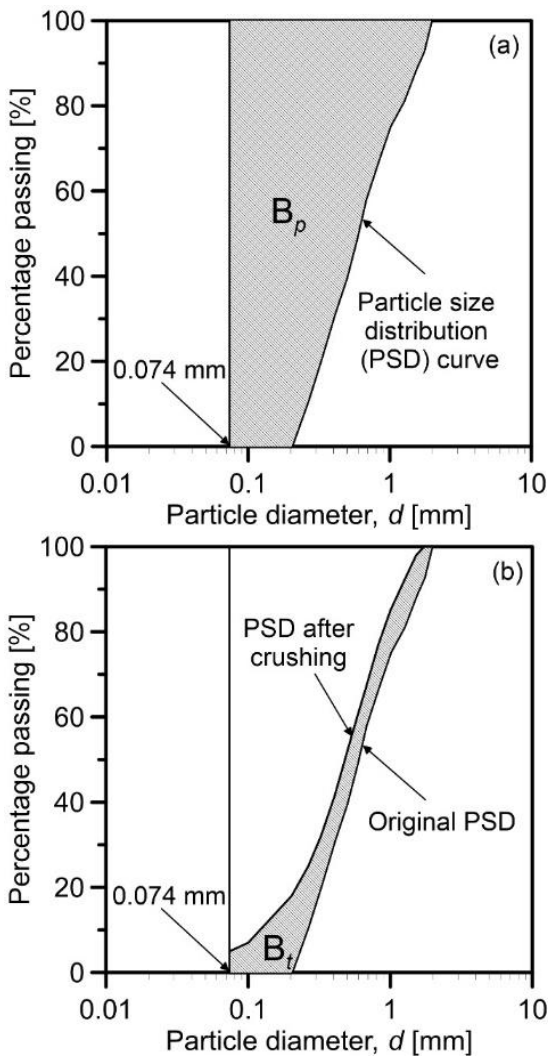

Fig. 1. Hardin's [8] breakage parameters. (a) Breakage potential $\left(B_{p}\right)$, and (b) total breakage $\left(B_{t}\right)$

The breakage potential $\left(B_{p}\right)$ is equal to the area between the line defining the upper limit of the silt size $(d=0.074 \mathrm{~mm})$, and the part of the PSD for which $d>$ $0.074 \mathrm{~mm}$. Its value is easily quantified by calculating the area in Fig. 1(a). If the soil in Fig. 1(a) is subject to crushing, the position and shape of the PSD may change. Hence, the total breakage is calculated by quantifying the area in Fig. 1(b). The ratio between the total breakage $\left(B_{t}\right)$ and the breakage potential $\left(B_{p}\right)$ is defined as relative breakage, $B_{r}\left(=B_{t} / B_{p}\right)$.

Note that the definition of $B_{r}$ requires two reference states; the initial PSD (characterised by $B_{p}$ ) and the final/limit PSD (assuming that the soil will crush until all particles have a size $\mathrm{d}=0.074 \mathrm{~mm}$ ). According to more recent studies (e.g. [5]) the limit grading is considered to be a fractal PSD and often associated to a comminution limit (e.g. [9, 10]). These findings are not ignored within existing studies using grading entropy coordinates. For example, the work in [13] recognises that the final state of the crushing tests can be characterized by the fractal distribution.

\subsection{Grading entropy coordinates - Lörincz (1986)}

Although grading entropy draws from the concept of statistical entropy, a distinction should be made between them. The statistical entropy of a thermal distribution is concerned with the summation of probabilities around achieving equivalent configurations in gas molecules-in order for energy be transferred efficiently. This definition is not applicable to (comparatively larger) soil particles. However, in grading entropy a similarity is shared between the calculation of statistical probabilities for particle configurations in a given sieve fraction, which is given as the relative frequency, $x_{i}$. Within this context and considering that a more extensive treatment can be found elsewhere, the mathematical definitions of the grading entropy coordinates are simply noted here. The coordinate pair $\left(S_{0}, \Delta S\right)$ that defines the grading entropy coordinates is given by the base entropy $\left(S_{0}\right)$ and entropy increment $\Delta S$, defined as:

$$
\begin{gathered}
S_{0}=\sum_{i=1}^{n} x_{i} S_{o i}, \\
\Delta S=-\frac{1}{\ln (2)} \sum_{i=1}^{n} x_{i} \ln x_{i},
\end{gathered}
$$

where $x_{i}$ is the relative frequency of the fraction $i$, $(i=1, \ldots, N)$, and $S_{0 i}$ is the eigen-entropy of the fraction $i,(i=1, \ldots, N)$. The eigen-entropy of fraction $(i)$ is defined here as an integer identifier for each of the sieves in a conventional sieving test as conventionally used for soil classification. The first fraction comprises the range from $2^{-22}$ to $2^{-21} \mathrm{~mm}$ with $S_{0}$ of 0 [11]. Earlier studies using grading entropy coordinates also consider a normalised version of the grading entropy coordinates $\left(S_{0}, \Delta S\right)$ which are normally referred to as the relative base entropy $(A)$, and the normalised entropy increment $(B)$, which also define a corresponding Cartesian coordinate pair $(A, B)$. For simplicity, the analyses presented in this paper is restricted to the nonnormalised grading entropy coordinates $\left(S_{0}, \Delta S\right)$. Note however that for certain practical applications, such as internal stability rules [11] used for filter design a mixed coordinate pair using normalised and non-normalised coordinate pair may be used (i.e. $[A, \Delta S]$ ). The key aspect of such coordinates is that the base entropy $(A$, or $S_{0}$ ) relates to mean particle size. In rigorous mathematical terms it is a logarithmic mean of the particle diameter and may be considered as a function of the mean particle size $\left(d_{50}\right)$. The bigger $d_{50}$, the bigger the base entropy $\left(A\right.$, or $\left.S_{0}\right)$. On the other hand, the entropy increment $(B$, or $\Delta S)$ is related to the conventional coefficients of curvature and uniformity (i.e. $c_{c}$ and $c_{u}$ ). Mathematically speaking, the entropy increment quantifies how a PSD is affected by the number of fractions/sieves. In other words, PSD's which are uniformly sized (and with mass retained across fewer sieves) have a low entropy increment. This is because a well-graded specimen retains material over a larger number of sieve sizes. Changes in grading entropy coordinates can also be understood in terms of 
simple PSD changes. A PSD with the same curvature but of smaller $d_{50}$ has a lower value of base entropy ( $A$, or $\left.S_{0}\right)$, and it will retain its entropy increment $(B$, or $\Delta S)$ as long as the number of sieves in which its mass is retained does not change by the corresponding size change. Similarly, two PSD curves with similar $d_{50}$ and different curvature (i.e. well-graded and uniform specimens with similar $d_{50}$, respectively) may have similar $\left(A\right.$, or $\left.S_{0}\right)$ and different $(B$, or $\Delta S)$ coordinates, respectively.

\section{Effect of PSD on particle breakage}
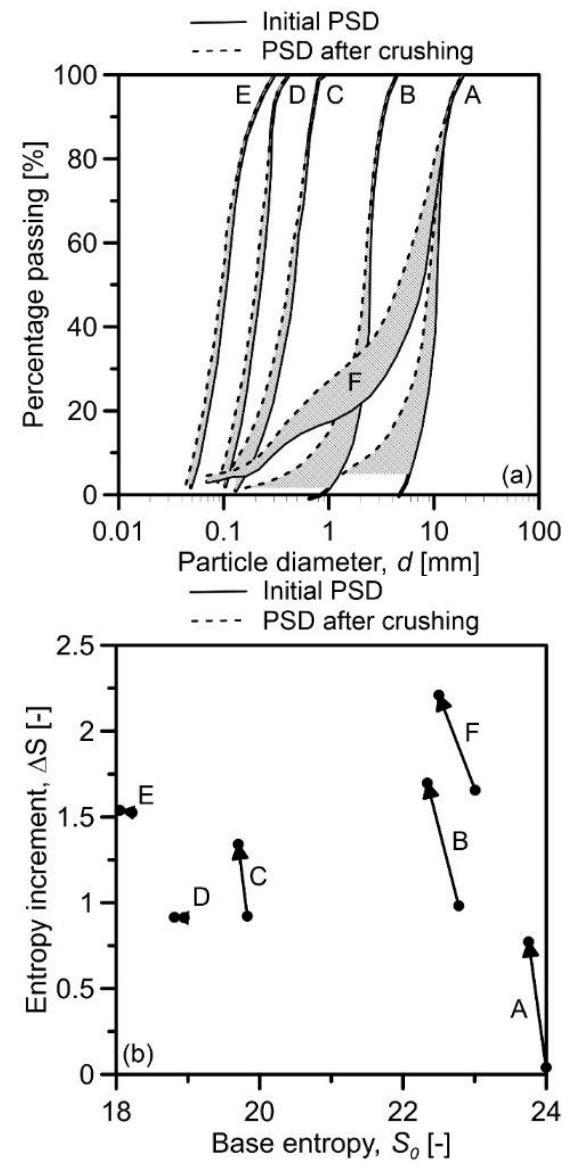

Fig. 2. a) PSD curves for crushed granite (modified after [17]); b) Same data in terms of entropy coordinates. Arrows indicate final/crushed states.

It is well established that the potential for breakage in a soil particle increases with its size. The probability of material defects also increases with particle size. Numerical research using particle methods (e.g. DEM) has widely recognised that stress transmission in granular materials is strongly heterogeneous and that strong force chains tend to form. In the case of wellgraded specimens, larger stresses tend to be carried by the largest particles, making them more prone to breakage. Breakage also depends on mineralogy. Carbonate sands are susceptible to particle breakage at stress levels much smaller than those required for quarzitic sands $[4,16]$.

Hardin [8] uses data by Lee and Farhoomand [17] to demonstrate that the total breakage $\left(B_{t}\right)$ for specimens of the same material, at a given void ratio, and loaded along a specified effective stress path to the same effective stress state is approximately proportional to the breakage potential $\left(B_{p}\right)$. This implies that relative breakage $\left(B_{r}\right)$ is independent of PSD. Whilst the same conclusion can be made using grading entropy coordinates, the focus is on how PSD changes are observed in terms of grading entropy coordinates. Fig. 2(a) shows the PSD data before and after crushing for granite specimens by [17] and Fig 2(b) illustrates the same data in terms of the grading entropy coordinates.

In Fig. 3(b) each line represents the grading entropy path for each specimen before and after crushing. For each case, the initial state is to the right of the crushed specimen (because the initial particle sizes are bigger). Note that specimens A and F have the largest (mean) particle size. Correspondingly, they have the largest values of base entropy $\left(\mathrm{S}_{0}\right)$, whilst specimens $\mathrm{D}$ and $\mathrm{E}$ are the smallest. The entropy paths are directed to the left because as crushing progresses the mean particle size reduces. As noted in Fig 2(a) the largest PSD change is experienced by specimens $\mathrm{A}$ and $\mathrm{F}$, as confirmed in Fig 2(b) by the longest entropy paths. The short paths for specimens D and E reflect their limited changes in PSD before and after crushing. Note however that specimen $\mathrm{C}$ experiences greater PSD changes after crushing than specimens D and E, respectively. In this case, a wider PSD is produced after crushing (with a larger range of particle sizes than for specimens $\mathrm{D}$ and E). Specimen C is located further to the right than these specimens due to its larger value of $d_{50}$. Such differences may not be as significant using other grading indices. This is an interpretation inferred from changes in entropy increment $(\Delta S)$. Specimens $A, B$ and $F$ experience changes in PSD shape (in addition to changes in particle sizes), hence they show greater changes in grading entropy coordinates. It follows that specimens D and E, which experience PSD changes in size, but insignificant changes in shape the are quantified as insignificant changes in entropy increment $\Delta S$.

To summarise, in contrast to existing approaches, grading entropy coordinates can help in quantifying the PSD evolution in terms of both size and shape. Furthermore, knowing the initial PSD state, a final state could be predicted. More evidence would of course help to further validate this statement.

\section{Effect of stress state on particle breakage (as a function of PSD)}

As discussed by Hardin [8] and other researchers, it can be expected that under extremely high stresses it would be possible for most (or all) particles in a soil specimen to be crushed to the extent that a fractal distribution may be obtained [e.g. 5, 7]. This intuitively implies that breakage increases with stress level.

Fig. 3 illustrates experimental data by [17], also used by [8] to illustrate the benefits of the relative breakage index $\left(B_{r}\right)$. For these tests, granite samples were subject to constant $R=\sigma_{1}^{\prime} / \sigma_{3}^{\prime}$ stress paths. The value of the breakage stress $\left(\sigma_{b}^{\prime}\right)$ was determined by [8] as a function of the normal and shear stresses. It is therefore akin to 
breakage limits like that defined by [6] and now commonly used by other researchers on the basis of more rigorous theories. With reference to Fig. 3(a), note that as the stress level increases the base entropy $\left(\mathrm{S}_{0}\right)$ decreases. This confirms that as the stress level increases the amount of breakage increases, and the mean particle size reduces. This coincides with the findings by [8] and other researchers. Fig. 3(b) provides some additional insight. As stress level increases the entropy increment also increases. This indicates that during crushing at high stress the PSD shape also changes. This change seems to be proportional to stress level. Interestingly, the work by Hardin [8] postulated the relative breakage being independent of stress path. Recent research [e.g. 2, 5, 6, 7] now convincingly defends that breakage is indeed dependent on both stress level and stress path. Fig. 3 illustrates that such dependencies are well captured by grading entropy coordinates.
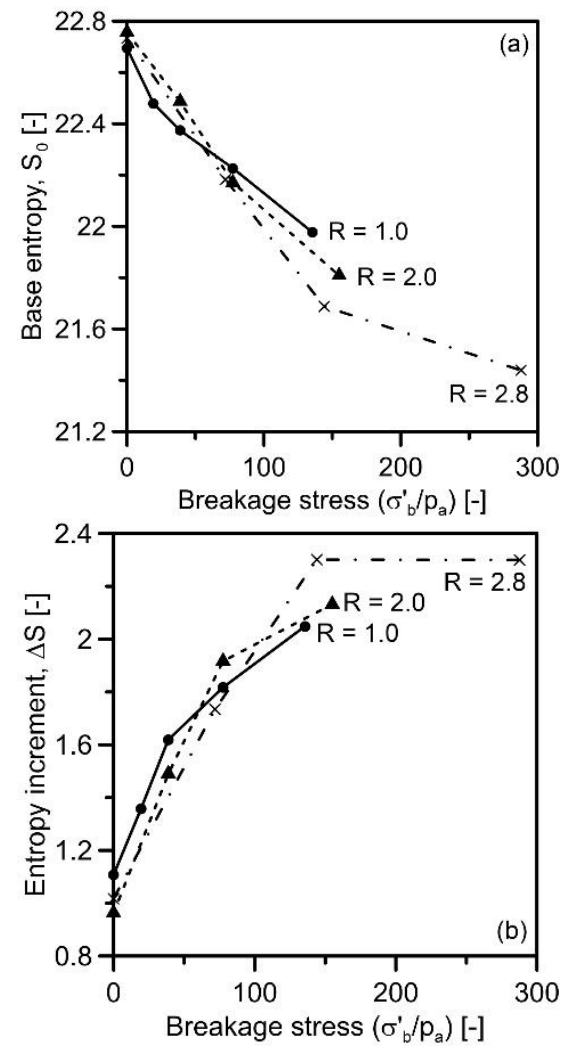

Fig. 3. Evolution of a) base entropy and b) entropy increment with breakage stress $\left(\sigma_{b}^{\prime}\right)$ - data for crushed granite by [17].

\section{Discussion and conclusions}

The previous sections have attempted to demonstrate that using grading entropy coordinates allows for a more accurate and potentially useful way to describe any PSD. It has been argued that grading entropy coordinates can capture well PSD changes during crushing. The physical meaning of the changes in the grading entropy coordinates was also discussed. Despite the limited data shown here, findings have shown that grading entropy coordinates provide some improvement in terms of parameter/index sensitivity. This has been shown by illustrating the evolution of breakage as a function of PSD, stress level and stress path.

Whilst it is also well recognised that breakage is affected by density (void ratio), particle shape, mineralogy, and particle hardness (which may be also affected by moisture content), the performance of grading entropy coordinates to quantify these effects are out of scope for this paper. It seems clear however that such parameters have great potential and further evaluation/assessments are ongoing.

\section{References}

[1] M.T. Manzari, Y.F. Dafalias, Géotechnique 47, 255-272 (1997)

[2] A.R. Russell, N. Khalili, Can. Geotech. J. 41, 1179-1192 (2004)

[3] G. Li, Y.J. Liu, C. Dano, P.Y. Hicher, J. Eng. Mech. 139, 606-615 (2013)

[4] M.R. Coop, D.W. Airey, Carbonate sands, in Characterisation and engineering properties of natural soils, Balkema, Rotterdam (2003)

[5] D. Muir Wood, K. Maeda, Acta Geotechnica 3, 3$14(2008)$

[6] J.P. de Bono, G.R. McDowell, Géotechnique 68, 575-589 (2018)

[7] M.O. Ciantia, M. Arroyo, C. O’Sullivan, A. Gens, T. Liu, Géotechnique 69, 1-15 (2019)

[8] B.O. Hardin, J. Geotech. Eng-ASCE 111, 11771192 (1985)

[9] I. Einav, J. Mech. Phys. Solids 55, 1274-1297 (2007)

[10] D. Muir Wood, Can. Geotech. J. 44, 1329-1350 (2007)

[11] J. Lörincz, Grading entropy of soils. Doctoral thesis, Technical Univ. of Budapest, Hungary. (1986)

[12] J. Lőrincz, E. Imre, M. Gálos, Q. P. Trang, K. Rajkai, S. Fityus, G. Telekes, Int. J. Geomech. 5, 311-319 (2005)

[13] J. Lőrincz, E. Imre, S. Fityus, P.Q. Trang, T. Tarnai, I. Talata, V.P. Singh, Entropy 17, 27812811 (2015)

[14] D. Barreto, J. Leak, V. Dimitriadi, J. McDougall, E. Imre, J. Lörincz, Grading entropy coordinates and criteria for evaluation of liquefaction susceptibility, in Earthquake Geotechnical Engineering for Protection and Development of Environment and Constructions, CRC Press, Rome (2019)

[15] J.R. McDougall, E. Imre, D. Barreto, D. Kelly, Géotechnique 63, 262-266 (2013)

[16] M.R. Coop, I.K. Lee, The behaviour of granular soils at elevated stresses, in Predictive soil mechanics, Thomas Telford, London (1993)

[17] K.L. Lee, I. Farhoomand, Can. Geotech. J. 4, 68$86(1967)$ 